\title{
Birefringence Upper Limit Analysis of Low Birefringence Fibers Employed in the Faraday Effect Current Sensors
}

\author{
N. Vukovic* ${ }^{\mathrm{a}}$, M. Segura Sermiento ${ }^{\mathrm{a}}$, T. May-Smith ${ }^{\mathrm{a}}$, W. H. Loh ${ }^{\mathrm{a}}$, M. N. Zervas ${ }^{\mathrm{a}}$ \\ ${ }^{\mathrm{a} O p t o e l e c t r o n i c s ~ R e s e a r c h ~ C e n t r e, ~ U n i v e r s i t y ~ o f ~ S o u t h a m p t o n, ~ S o u t h a m p t o n, ~ S O 17 ~ 1 B J, ~ U K ~}$
}

\begin{abstract}
The theoretical model of the Faraday rotation in the low birefringence optical fiber is proposed to serve as a convenient tool for the determination of the birefringence upper limit allowed to retain current sensor sensitivity. The measurement technique offers a fast and efficient determination of the ultra-low linear birefringence when other techniques are not sensitive enough or they are difficult to implement. A temperature dependence of the Faraday rotation and its causes are investigated.
\end{abstract}

Keywords: Optical sensing, Faraday effect current sensors, low birefringence measurement, fiber characterization

\section{INTRODUCTION}

Optical current sensors based on the Faraday effect have been widely investigated and still remain an active area of research $^{1-3}$. One popular approach is to use spun optical fibers, where by spinning the preform during fiber drawing a permanent twist is imparted which restores a circular symmetry, leading to the ultra-low linear birefringence ${ }^{4}$ of the order of $\sim 10^{-9}$. However, in spite of the negligible intrinsic linear birefringence, in practice linear birefringence still exists in current sensors, owing to the bend- and coating-induced stresses, and its presence quenches the Faraday effect ${ }^{5}$. In general, different approaches are used to minimize or eliminate this induced non-negligible birefringence, including annealing and twisting the fiber, as well as by utilizing spun elliptically birefringent fibers ${ }^{1,6}$. Although spun elliptically birefringent fibers are widely used as an industry standard current sensors, there is still interest in using spun low birefringence fibers as a platform for current sensing applications. Compared to its high-birefringence counterpart, spun low-birefringence fibers retain many of the advantageous properties such as ease of fabrication and low polarization mode dispersion for the applications beyond current sensors, into the telecommunications industry. However, to date, many fiber birefringence measurement techniques lack sensitivity and are not capable of measuring ultra low levels of birefringence. Typically, traditional measurement techniques are limited to the birefringence of $\sim 10^{-8}$, and require long fiber lengths. Therefore a development of a sensitive measurement technique remains a challenge.

In this paper we focus on a technique to provide an insight into the birefringence of the fibers, including spun low birefringence fibers. Using a simple Faraday effect current sensor experiment and a corresponding theoretical model of the Faraday rotation in a birefringent fiber we show that this can be used to infer the intrinsic birefringence of the fiber. In particular, the upper limit of the total birefringence that is permitted to retain sensor sensitivity can be determined. This method is simple, fast and requires short lengths of fiber, and is potentially applicable to a wide variety of fibers. An account of the thermal properties of the Faraday effect in low-birefringence fibers will be given, demonstrating the detrimental effect of the fiber coating to the sensor temperature dependence.

\section{FARADAY EFFECT CURRENT SENSOR CONFIGURATION}

Our investigations are based on $\mathrm{SiO}_{2}$ single mode commercial and in-house made fibers. The experimental setup used for the Faraday rotation and its temperature dependence measurement is shown in Fig. 1. The output light from a laser diode at $1550 \mathrm{~nm}$ linearly polarized in the $\mathrm{x}$ direction is launched through a polarization insensitive beam splitter into the fiber. The fiber is put straight along the axis of $\sim 1 \mathrm{~m}$ long hollow solenoid with four 1400-turns coils of copper wire, driven by the DC power source with the maximum current of 4A. The fiber end is butt-coupled to the gold coated mirror where light is reflected and passed back to the polarization insensitive beam splitter, through the Wollaston prism which separates the two orthogonal polarizations and has the transmission axis fixed at $45^{\circ}$ with respect to the input.

*ntv@orc.soton.ac.uk; phone +44 (0) 23 80593141; fax +44 (0) 23 80593142; soton.ac.uk 
Two Germanium photodetectors with an identical intensity response measure the power of each beam. Due to the use of a mirror and owing to the non-reciprocal nature of the Faraday effect and the reciprocity of linear birefringence ${ }^{1}$, the Faraday rotation is doubled and some unwanted perturbations, including the unwanted residual circular birefringence ${ }^{1}$, are cancelled.

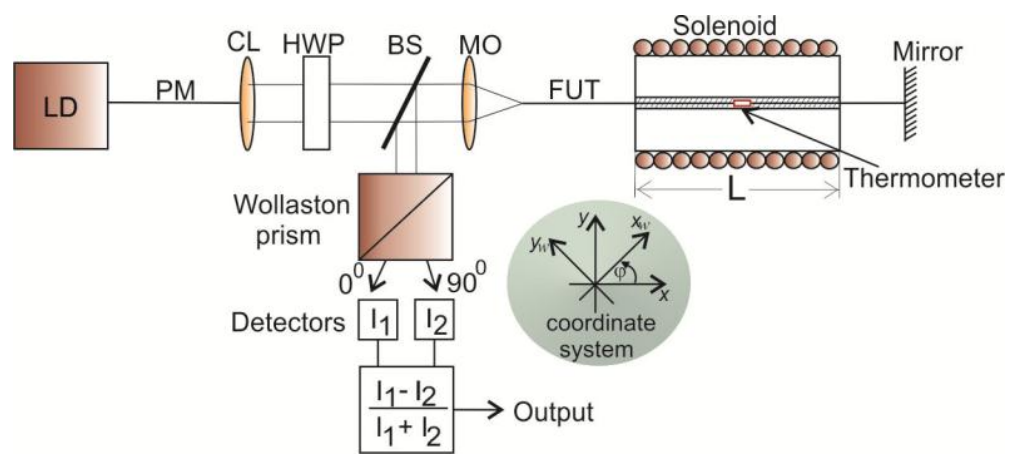

Figure 1. Schematic of the Faraday current sensor experimental setup (LD -Laser Diode, PM - Polarization Maintaining Fiber, HWP - Half Wave Plate, CL - Collimating Lens, BS - Polarization-insensitive Beam Splitter, MO - Microscope Objective, FUT - Fiber Under Test). Inset: Coordinate system $-x$ and $y$ are principal axes of birefringence in the fiber and $x_{w}$ and $y_{w}$ are transmission axes of the Wollaston prism.

The Faraday effect is produced from the interaction of magnetic field and light in a current carrying conductor wrapped around a single mode fiber, and the Faraday induced polarization rotation is linearly proportional to the current. In the ideal situation, when there is no linear birefringence in the fiber, the Faraday rotation angle $\left(\theta_{\text {ideal }}\right)$ is proportional to the Verdet constant $(V)$ of the fiber, the applied magnetic field $(B)$, i.e. the current $(I)$ and the interaction length $(L)$, according to the relation $\theta_{\text {ideal }}=V B L=\mu_{0} N V I$, where $\mu_{0}=4 \pi \times 10^{-7} \mathrm{~N} / \mathrm{A}^{2}$ is magnetic permeability of free space, $\mathrm{B}=\mu_{0} n I$ is the applied magnetic field, $N$ is the number of turns of wire, and $n=N / L$ is a turns density.

In the case of non-negligible linear birefringence of the fiber, the Faraday rotation angle can be expressed using the integral approach of the Ampere's law for the magnetic field, in the following way ${ }^{3,6}$ :

$$
\theta_{\text {non-ideal }}=V \int_{0}^{L} B(z) d z=\frac{V B}{\Delta \beta} \sin (\Delta \beta \cdot L)=V B L \sin c(\Delta \beta \cdot L),
$$

where $\Delta \beta=2 \pi \Delta n / \lambda$ is the intrinsic linear birefringence, $\Delta n=n_{x}-n_{y}$ is the difference of refractive indices for the two polarization directions, and $\lambda$ is the wavelength of light. The Verdet constant of the silica fiber used in the experiment is $0.54 \mathrm{rad} /(\mathrm{Tm})$ at $1550 \mathrm{~nm}$. The variation of the Verdet constant with the temperature is negligible $(d V / d T) / V=6.9 \times 10^{-5}$ $\mathrm{K}^{-1}$. The optical arrangement shown can be described by Jones calculus and the intensities transmitted by Wollaston prism can be obtained by $I_{1}=\cos ^{2}(\varphi-2 \theta)$ and $I_{2}=\sin ^{2}(\varphi-2 \theta)$, where $\varphi$ is the angle of orientation of the Wollaston prism with respect to the axes $x$ and $y$ (Fig.1). The output response produced from the measurement system is $\mathrm{S}=\left(\mathrm{I}_{1}-\mathrm{I}_{2}\right) /\left(\mathrm{I}_{1}+\mathrm{I}_{2}\right)=\cos 2 \varphi \cos 4 \theta+\sin 2 \varphi \sin 4 \theta$. When $\varphi=45^{\circ}, \mathrm{S}$ attains maximum sensitivity and the Faraday rotation is given by $\mathrm{S}=\sin 4 \theta$, allowing for the measurement of the Faraday rotation angle $\theta$.

\section{LINEAR BIREFRINGENCE DETERMINATION}

In order to characterize the fiber linear birefringence, firstly we simulated the Faraday rotation after the propagation distance of $1 \mathrm{~m}$, in accordance to the model of non-negligible birefringence of the fiber given by Eq.(1), and the results are shown in Fig.2. Fig.2(a) shows that the transfer of power between the orthogonal axes of the birefringent fiber is maximum after a quarter of the polarization beat length, and reduces to zero over half of the beat length; intrinsic linear birefringence as labeled on the right-hand-side of the figure. The contour plot in Fig. 2(b) shows the maximum rotation of $\sim 0.4^{\circ}$, which is obtained at $1 \mathrm{~m}$ length of the fiber and the linear birefringence of $\sim 10^{-9}$. Fig.2(c) shows the normalized Faraday rotation angle as a function of linear birefringence and it can be observed that the maximum rotation is achieved when the linear birefringence is less than $10^{-7}$. The sensitivity of the Faraday rotation angle to the linear birefringence $\Delta \mathrm{n}$ is calculated and shown in Fig. 2(d) with the point of maximum sensitivity calculated for $\Delta \mathrm{n} \sim 2.5 \times 10^{-7}$. Significantly, this analysis provides justification why conventional single mode fibers are not good candidates for the current sensors application because their birefringence is $\sim 10^{-7}$ and at this value of linear birefringence they are extremely sensitive to 
environmental, mechanical and temperature effects that affect fiber linear birefringence, as shown in Fig. 2(d). This result explains the motivation to use the ultra-low birefringence fibers for the Faraday effect current sensors. Owing to the sensitivity of the Faraday rotation to $\Delta \mathrm{n}$, it can be generally considered that the birefringence larger than $\sim 10^{-7}$ greatly reduces Faraday rotation and increases sensitivity to unwanted perturbations.
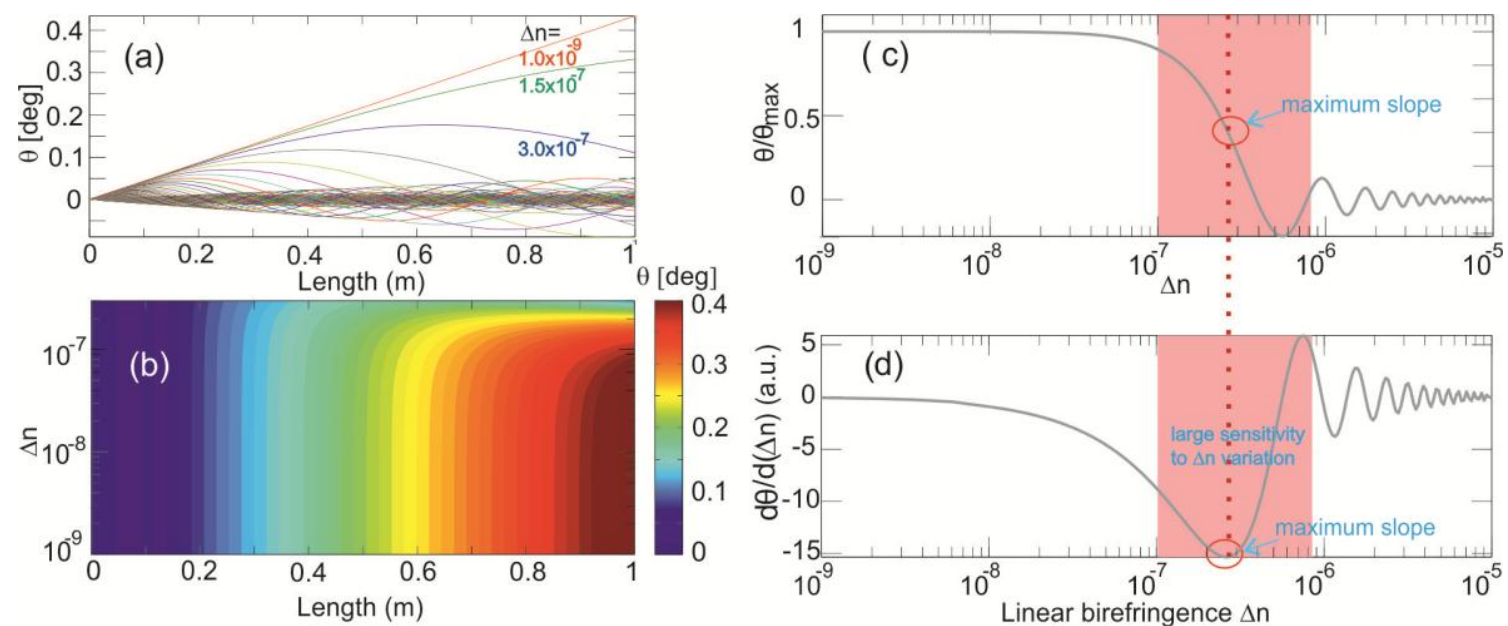

Figure 2. (a) Faraday rotation angle $\theta$ as a function of fiber linear birefringence and length for $\mathrm{I}=4 \mathrm{~A}$ and (b) corresponding contour plot. (c) Normalized Faraday rotation angle versus $\Delta \mathrm{n}$ and (d) sensitivity of the Faraday rotation angle to $\Delta \mathrm{n}$, for $\mathrm{L}=1 \mathrm{~m}$ and $\mathrm{I}=4 \mathrm{~A}$.

Next, we explore how this simple experimental setup can be efficiently used for the determination of intrinsic birefringence of low birefringence fibers. To characterize the linear birefringence, we took the measurements of the Faraday rotation using the experimental setup shown in Fig.1 for different values of DC current in a range 0-4A. We compared the experimental results with the simulations and we used the best fit to estimate the linear birefringence of the fiber. Importantly, the precautionary measures are taken to avoid all sources of stress induced birefringence in the setup due to fiber mounting, so we can assume that only fiber intrinsic birefringence is present. Thus, under this assumption, the results of the experiment and the simulation are shown in Fig.3. The best fit to the simulation at room temperature $\left(23^{\circ} \mathrm{C}\right)$ indicates that the fiber birefringence is $\sim 2.2 \times 10^{-7}$, which implies that our fiber lies at the point of nearly maximum sensitivity to a linear birefringence and thus a significant variation from the ideal theoretical value of Faraday rotation angle is observed. In order to justify and validate our results we have conducted an independent measurement using a standard polarizer-analyzer technique on a commercial single mode fiber and on an in-house fabricated fiber used in Fig. 3 and we have obtained an excellent agreement between the two techniques. This characterization method is potentially useful in ultra-low birefringence measurement and could be used as a fast way for the determination of the intrinsic birefringence at short lengths of the low birefringence fabricated fibers.

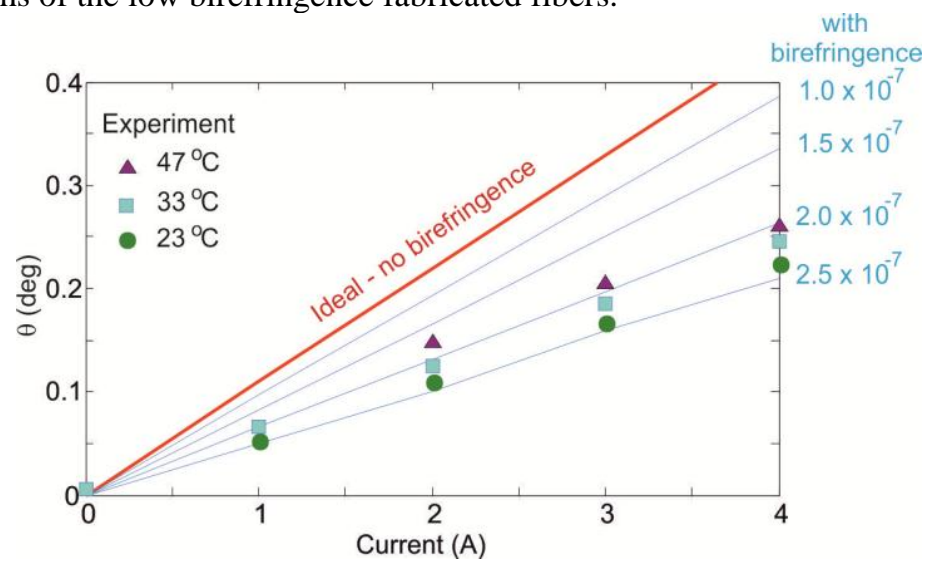

Figure 3. Simulation and experimental results of the Faraday rotation for $1 \mathrm{~m}$ - long coated in-house fabricated silica single mode fiber (A) and $\mathrm{I}=4 \mathrm{~A}$. 
In addition, the experimental setup shown in Fig. 1 was used to carry out the measurement of the Faraday rotation versus temperature at different temperatures up to $\sim 65^{\circ} \mathrm{C}$ for two different in-house fabricated silica single mode fibers labeled as A and B. Fig. 4(a) shows the temperature dependence measurements for the coated and uncoated fiber (A). It can be observed that for the coated fiber the Faraday rotation increases with the increasing temperature. This trend corresponds to a similar observation in Ref. (1) and we anticipate that it is due to the drop of the viscosity of the optical fiber coating during the temperature increase, thus reducing the coating induced linear birefringence. In the case of the uncoated fiber, Fig. 4(a) shows nearly constant values of Faraday rotation, indicating that the fiber coating is a root cause of the Faraday effect temperature dependence. This experimental result agrees with the theoretical prediction shown in Fig. 2(d) that for the intrinsic birefringence of $\sim 2.2 \times 10^{-7}$ there is a large sensitivity to the linear birefringence variation. Fig. 4(b) shows Faraday rotation as a function of temperature for the in-house fabricated fiber (B) when current is switching from 0A to $4 \mathrm{~A}$, and it indicates that the birefringence of this fiber is sensitive to temperature variation, too. We anticipate that this coating induced temperature dependence is due to the temperature induced radial stress and axial strain in the fiber through the fiber coating. Further investigations, including the attempts to anneal the fibers and release the built in strain, of the effect of fiber coating are performed on different fibers and they are currently on-going.
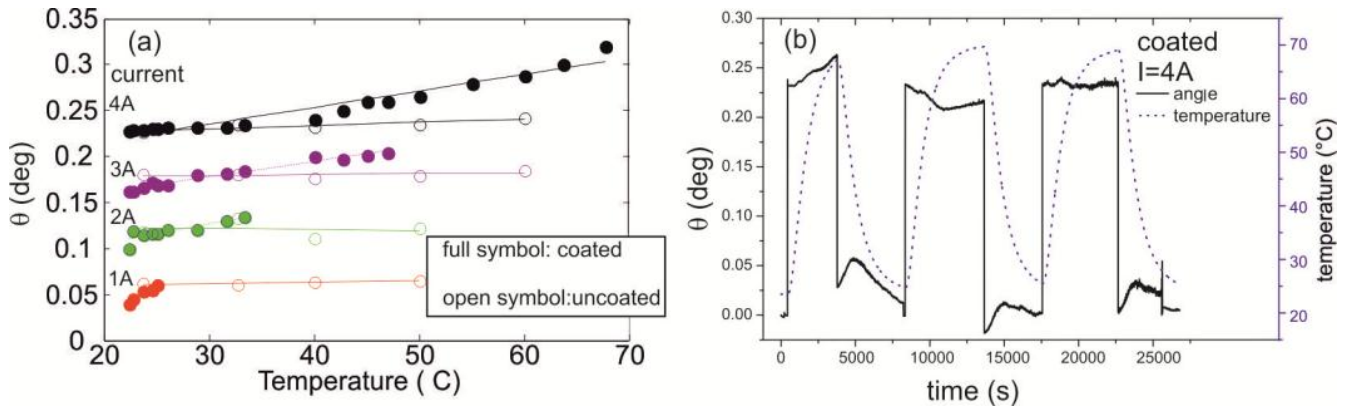

Figure 4. (a) Temperature dependence of the Faraday rotation for the coated fiber and uncoated in-house fabricated fiber (A).

(b) Faraday rotation as a function of temperature for coated in-house fabricated fiber (B) when current is switching from 0A to 4A.

\section{CONCLUSION}

In conclusion, we propose a simple approach in which we built the Faraday effect measurement setup and applied theoretical model to infer the linear birefringence of the fibers. The key advantage of this characterization method is that it employs only a meter-length of fiber and can be used for ultra-low birefringence fibers where conventional birefringence measurements are difficult to perform. Importantly, we theoretically determine the sensitivity of the Faraday rotation to the intrinsic birefringence and show why low birefringent fibers with $\Delta \mathrm{n}$ greater than $10^{-7}$ are not adequate for current sensors applications. We anticipate that this method could provide guidelines for the linear birefringence upper limit determination for the fibers employed in the Faraday effect current sensors. In addition, we present preliminary measurement results which show fibers sensitivity to temperature variation and we discuss the means to resolve this issue.

\section{REFERENCES}

[1] Laming, R. I. and Payne, D. N., "Electric current sensors employing highly birefringent optical fibers," J. Lightwave Technol. 7(12), 2084-2094 (1989).

[2] Ren, Z., Wang, Y. and Robert, P. A., "Faraday rotation and its temperature dependence measurements in lowbirefringence fibers," J. Lightwave Technol. 7(8), 1275-1278 (1989).

[3] Chen, G. Y., Brambilla, G. and Newson, T. P., "Birefringence treatment of non-ideal optical microfiber coils for continuous Faraday rotation," Electron. Lett. 49(11), 714-715 (2013).

[4] Payne, D. N., Barlow, A. J. and Ramskoy-Hansen, J. J., "Development of low- and high- birefringence optical fibers," IEEE T. Microw. Theory MTT-30(4), 323-334 (1982).

[5] Cruz, J. L., Andres, M. V. and Hernandez, M. A., "Faraday effect in standard optical fibers: dispersion of the effective Verdet constant," Appl. Opt. 35(6), 922-927 (1996).

[6] Rose, A. H., Ren, Z. B. and Day, G. W., "Twisting and annealing optical fiber for current sensors," J. Lightwave Technol. 14(11), 2492-2498 (1996). 